\title{
On transcendental automorphisms of algebraic foliations
}

by

\author{
B. Scárdua (Rio de Janeiro)
}

\begin{abstract}
We study the group $\operatorname{Aut}(\mathcal{F})$ of (self) isomorphisms of a holomorphic foliation $\mathcal{F}$ with singularities on a complex manifold. We prove, for instance, that for a polynomial foliation on $\mathbb{C}^{2}$ this group consists of algebraic elements provided that the line at infinity $\mathbb{C} P(2) \backslash \mathbb{C}^{2}$ is not invariant under the foliation. If in addition $\mathcal{F}$ is of general type (cf. [20]) then $\operatorname{Aut}(\mathcal{F})$ is finite. For a foliation with hyperbolic singularities at infinity, if there is a transcendental automorphism then the foliation is either linear logarithmic, Riccati or chaotic (cf. Definition 1). We also give a description of foliations admitting an invariant algebraic curve $C \subset \mathbb{C}^{2}$ with a transcendental foliation automorphism.
\end{abstract}

1. Introduction and main results. In this paper we study the group of (self) isomorphisms of a foliation. Given a codimension one holomorphic foliation $\mathcal{F}$ with singularities on a complex manifold $M$ we denote by $\operatorname{Aut}(\mathcal{F})$ the maximal subgroup of $\operatorname{Aut}(M)$ whose elements preserve the foliation $\mathcal{F}$. This object has been studied in [20] where it is proven that $\operatorname{Aut}(\mathcal{F})$ is finite for $\mathcal{F}$ of general type on a (compact) projective surface. We recall (cf. [20]) that a foliation $\mathcal{F}$ on a projective surface $M^{2}$ is of general type if its Kodaira dimension (cf. [17]) is equal to 2 . Our results extend in a way the result above to open algebraic surfaces. Indeed, we are concerned with the non-compact case and its applications to the classification of holomorphic flows on $\mathbb{C}^{2}$, for instance. Our first result is the following:

Proposition 1. Let $\mathcal{F}$ be a foliation with hyperbolic singularities on $\mathbb{C} P(2)$. Suppose there exists some affine open subset $U \subset \mathbb{C} P(2)$ such that the restriction $\left.\mathcal{F}\right|_{U}$ contains a holomorphic flow in its group of automorphisms. Then $\mathcal{F}$ is, in suitable affine coordinates, a linear hyperbolic foliation.

By an affine set $U \subset \mathbb{C P}(2)$ we mean the complement of an algebraic curve $\Gamma \subset \mathbb{C} P(2)$. This preliminary result suggests that the complexity of the transverse structure and that of the tangent structure are, in a certain

2000 Mathematics Subject Classification: 37F75, 32S65. 
sense, in inverse proportion. This idea is enforced by our next results. The first is the following extension lemma:

LEMma 1 (Extension Lemma). An entire automorphism $\varphi: \mathbb{C}^{2} \rightarrow \mathbb{C}^{2}$ of an algebraic foliation $\mathcal{F}$ on $\mathbb{C P}(2)$ is algebraic provided that the infinity $\mathbb{P}_{\infty}^{1}=\mathbb{C} P(2) \backslash \mathbb{C}^{2}$ is not invariant under $\mathcal{F}$.

The above result actually holds for bimeromorphic maps $\varphi: \mathbb{C}^{2} \backslash \Gamma \rightarrow$ $\mathbb{C}^{2} \backslash \Gamma$ if we replace $\mathbb{P}_{\infty}^{1}$ by an irreducible (non-invariant) algebraic curve $\Gamma \subset \mathbb{C} P(2)$, as will be clear from the proof we give. We point out that a foliation automorphism $\varphi \in \operatorname{Aut}\left(\mathbb{C}^{2}\right)$ may extend to a bimeromorphic nonlinear map of $\mathbb{C} P(2)$ as is the case for $\mathcal{F}: d x=0$ and $\varphi(x, y)=\left(x, y+x^{n}\right)$. Denote by $\operatorname{Bim}(\mathcal{F})$ the group of bimeromorphic maps $\Phi: M \rightarrow M$ which preserve $\mathcal{F}$ in $M$ (these maps take leaves of $\mathcal{F}$ onto leaves of $\mathcal{F}$ wherever defined). Combining the extended Lemma 1 with Theorem 1 of [20] we immediately obtain:

COROLlaRY 1. Let $\mathcal{F}$ be a codimension one singular holomorphic foliation on $\mathbb{C P}(2)$ and $\Gamma \subset \mathbb{C P}(2)$ a non-invariant algebraic curve. Then we have natural group immersions $\operatorname{Bim}\left(\left.\mathcal{F}\right|_{\mathbb{C} P(2) \backslash \Gamma}\right) \subset \operatorname{Bim}(\mathcal{F}) \subset \operatorname{Bim}(\mathbb{C} P(2))$. In particular, $\operatorname{Bim}\left(\left.\mathcal{F}\right|_{\mathbb{C} P(2) \backslash \Gamma}\right)$ is finite if $\mathcal{F}$ is generic.

For a foliation $\mathcal{F}$ on a projective surface $M$ admitting a minimal model $\mathcal{G}$ on $\widetilde{M}$ (cf. [6]), we have a natural group isomorphism $\operatorname{Bim}(\mathcal{F}) \simeq \operatorname{Aut}(\mathcal{G})[20]$. Foliations without minimal model are listed as: rational fibrations, nontrivial Riccati foliations and the exceptional foliation $\mathcal{H}$ in [6] (see also Example 1.3 in [10]). In particular, if $\mathcal{F}$ is generic then it admits a minimal model $([20])$. In $[10]$ it is proved that for a foliation $\mathcal{F}$ on a projective surface if $\operatorname{Aut}(\mathcal{G}) \subsetneq \operatorname{Bim}(\mathcal{G})$ for every birational model $\mathcal{G}$ of $\mathcal{F}$ then we have the following possibilities: $\mathcal{F}$ is a rational fibration or $\mathcal{F}$ is the exceptional foliation $\mathcal{H}$ of [6], i.e., the foliation in Example 1.3 of [10]. Combining these results with Corollary 1 above we obtain:

COROLlaRY 2. Let $\mathcal{F}$ be a codimension one singular holomorphic foliation on $\mathbb{C} P(2)$ and $\Gamma \subset \mathbb{C} P(2)$ a non-invariant algebraic curve. Suppose that $\operatorname{Bim}\left(\left.\mathcal{F}\right|_{\mathbb{C} P(2) \backslash \Gamma}\right)$ is not finite. Then $\mathcal{F}$ is either a linear foliation, an elliptic fibration, a rational fibration, a Riccati foliation, or (birationally equivalent to) the exceptional foliation $\mathcal{H}$ of [6].

Proof. If $\mathcal{F}$ has a minimal model then we may assume that $\mathcal{F}$ is minimal and therefore $\operatorname{Aut}(\mathcal{F})=\operatorname{Bim}(\mathcal{F})$ is infinite. Theorem 1.1 of [10] then shows that $\mathcal{F}$ is either an elliptic fibration, a Riccati foliation or given by a global holomorphic vector field on $\mathbb{C} P(2)$, in which case $\mathcal{F}$ is necessarily linear. Now we may assume that $\mathcal{F}$ has no minimal model. Therefore, according to the above discussion $\mathcal{F}$ is either a non-trivial Riccati foliation, a rational 
fibration or the foliation $\mathcal{H}$ of [6] which is the foliation in Example 1.3 of [10].

For the transcendental case we shall be more precise by introducing a dynamical concept:

Definition 1 (chaotic foliation). We shall say that a foliation $\mathcal{F}$ on $\mathbb{C} P(2)$ which leaves invariant the line at infinity is chaotic if:

(ch-1) $\left.\mathcal{F}\right|_{\mathbb{C}^{2}}$ has no parabolic leaf (in the sense of potential theory, cf. [4]);

(ch-2) the foliation $\left.\mathcal{F}\right|_{\mathbb{C}^{2}}$ admits no (non-trivial) holonomy invariant measure, indeed the leaves of $\left.\mathcal{F}\right|_{\mathbb{C}^{2}}$ have exponential growth for the Fubini-Study metric ([9]);

(ch-3) the holonomy group of the leaf $L_{\infty}=\mathbb{P}_{\infty}^{1} \backslash\left(\operatorname{sing}(\mathcal{F}) \cap \mathbb{P}_{\infty}^{1}\right)$ is nonsolvable (see [19]);

(ch-4) all leaves of $\mathcal{F}$, except for $L_{\infty}$, are dense in $\mathbb{C} P(2)$ ([15], [12]).

$\left(\right.$ ch-5) $\left.\mathcal{F}\right|_{\mathbb{C}^{2}}$ (and therefore $\mathcal{F}$ on $\mathbb{C P}(2)$ ) is ergodic ([12]) and topologically rigid ([15], [23]).

Using this terminology we state our main results as follows:

Theorem 1. Let $\mathcal{F}$ be an algebraic foliation on $\mathbb{C} P(2)$ such that $\operatorname{sing}(\mathcal{F})$ $\cap \mathbb{P}_{\infty}^{1}$ is hyperbolic and $\operatorname{Aut}\left(\left.\mathcal{F}\right|_{\mathbb{C}^{2}}\right)$ contains a transcendental element $\varphi$ : $\mathbb{C}^{2} \rightarrow \mathbb{C}^{2}$. We have the following possibilities: (i) $\mathcal{F}$ is chaotic; (ii) $\mathcal{F}$ is a linear logarithmic foliation; (iii) $\mathcal{F}$ is a Riccati foliation.

Examples of foliations of logarithmic and Riccati type with transcendental foliation automorphisms are given in $\S 4$. Also, consider the following construction: let

$$
\varphi(x, y)=\left(x, \frac{y}{\alpha(x) y+\beta(x)}\right)
$$

for entire functions $\alpha, \beta \in \mathcal{O}(\mathbb{C})$. We can obtain examples of $\varphi$-invariant Riccati equations of the form $\mathcal{F}:\left[\left(x-a_{1}\right) \ldots\left(x-a_{r}\right)\right] d y-\left[y^{2} A(x)+y B(x)\right] d x$ $=0$, where in the affine system $(x, y) \in \mathbb{C}^{2} \subset \mathbb{C} P(2)$ we take the line at infinity to be $\mathbb{P}_{\infty}^{1}=\overline{(x=\infty)}$. It is then easy to obtain such examples with transcendental $\varphi$ and $\operatorname{sing}(\mathcal{F}) \cap \mathbb{P}_{\infty}^{1}$ hyperbolic. A linear hyperbolic foliation $\mathcal{F}: x d y-\lambda y d x=0, \lambda \in \mathbb{C} \backslash \mathbb{R}$, admits no transcendental foliation automorphisms (cf. Example 2).

As an immediate consequence of Theorem 1 we obtain:

Corollary 3. Let $\mathcal{F}$ be a foliation on $\mathbb{C} P(2)$ with hyperbolic singularities in $\mathbb{P}_{\infty}^{1}$ and such that $\operatorname{Aut}\left(\left.\mathcal{F}\right|_{\mathbb{C}^{2}}\right)$ contains a transcendental element. Suppose $\mathcal{F}$ has no algebraic invariant curve $C \subset \mathbb{C}^{2}$. Then $\mathcal{F}$ is chaotic.

In general, for a foliation admitting an algebraic invariant curve and with a transcendental automorphism we have: 
TheOREM 2. Let $\mathcal{F}$ be an algebraic foliation on $\mathbb{C} P(2)$ such that $\operatorname{Aut}\left(\left.\mathcal{F}\right|_{\mathbb{C}^{2}}\right)$ contains a transcendental element $\varphi: \mathbb{C}^{2} \rightarrow \mathbb{C}^{2}$. Suppose that $\mathcal{F}$ has an invariant algebraic curve $C \subset \mathbb{C}^{2}$. Then, up to replacing $\varphi$ by some power $\varphi^{n}, n \in \mathbb{N}$, we have (only) the following possibilities:

(tr.1) $\varphi(C)$ is not algebraic and $\left.\mathcal{F}\right|_{\mathbb{C}^{2}}$ is holomorphically equivalent to dy $=0$ in $\mathbb{C}^{2}$. Up to some entire automorphism taking $\left.\mathcal{F}\right|_{\mathbb{C}^{2}}$ to $d y=0$ we have $\varphi(x, y)=\left(a_{0}(y) x+a_{1}(y), b_{0}+b_{1} y\right)$ with $a_{0}(y), a_{1}(y) \in \mathcal{O}\left(\mathbb{C}^{2}\right), b_{0}, b_{1} \in$ $\mathbb{C}$ and some natural restrictions on the coefficients. In particular $C$ is isomorphic to the affine line, $C \simeq \mathbb{C}$.

(tr.2) There is $n \in \mathbb{N}$ such that $\varphi^{n}(C)=C$ in $\mathbb{C}^{2}$, and $C$ is, up to a polynomial automorphism of $\mathbb{C}^{2}$, in the following list:

(a) $\alpha(x)+y \beta(x)=0, \alpha(x), \beta(x) \in \mathbb{C}[x]$;

(b) a union of irreducible components of fibers of $x^{n} y^{m}, n, m \in \mathbb{N}$, $\langle n, m\rangle=1$

(c) a union of irreducible components of fibers of a polynomial $R(x, y)$ $=x^{n}\left(x^{l} y+P(x)\right)^{m}$ with $n, m, l \in \mathbb{N},\langle n, m\rangle=1, P(x) \in \mathbb{C}[x]$ of degree at most $l-1, P(0) \neq 0$.

In particular, $\bar{C}$ is rational and $\bar{C} \cap \mathbb{P}_{\infty}^{1} \leq 2$, so that each irreducible component $C_{0} \subset C$ satisfies $C_{0} \simeq \mathbb{C}$ or $\mathbb{C} \backslash\{0\}$.

(tr.3) $\varphi^{n}(C)$ is algebraic for some $n \in \mathbb{N}$ but $\varphi^{m}(C) \neq C$ for all $m \in \mathbb{N}$. In this case $\mathcal{F}$ admits a rational first integral $R: \mathbb{C P}(2) \rightarrow \mathbb{P}^{1}$; in particular all the leaves of $\mathcal{F}$ are algebraic.

Examples are given and we believe that one may go further in the discussion of Case (tr.2) above.

\section{The case of flows-Proof of Proposition 1}

2.1. Motivations and the compact case. Let $\mathcal{F}$ be a codimension $k$ foliation on a manifold $M$ with $\operatorname{singular} \operatorname{set} \operatorname{sing}(\mathcal{F})$. The group of foliation automorphisms of $\mathcal{F}$ is the subgroup $\operatorname{Aut}(\mathcal{F})$ of $\operatorname{Aut}(M)$ whose elements $\psi: M \rightarrow M$ satisfy $\psi(\operatorname{sing}(\mathcal{F}))=\operatorname{sing}(\mathcal{F})$ and $\psi^{*} \mathcal{F}=\mathcal{F}$, in other words, $\psi$ fixes the singular set and takes (non-singular) leaves of $\mathcal{F}$ onto (non-singular) leaves of $\mathcal{F}$. If $M$ is (complex) compact then $\operatorname{Aut}(M)$ is a (complex) Lie group, and since $\operatorname{Aut}(\mathcal{F})$ is a closed subgroup of $\operatorname{Aut}(M)$, it is a (complex) Lie subgroup. Thus we may consider the following general situation: $\operatorname{Aut}(\mathcal{F})$ is a complex Lie group and therefore there exists a Lie group action $\varphi: G \times$ $M \rightarrow M$ such that each automorphism $\varphi_{g}, g \in G$, belongs to $\operatorname{Aut}(\mathcal{F})$. We have two main (antipodal) cases: (i) $\varphi$ is transverse to $\mathcal{F}$; (ii) $\varphi$ is tangent to $\mathcal{F}$. In the rest of this section we shall consider the two main cases above in the compact two-dimensional situation for singular foliations. 
1. Let $\mathcal{F}$ be a singular codimension one foliation on a complex surface $M$ and assume there is a flow $\varphi: \mathbb{C} \times M \rightarrow M$ such that $\varphi_{t} \in \operatorname{Aut}(\mathcal{F})$ for all $t \in \mathbb{C}$. If $\varphi$ is transverse to $\mathcal{F}$ then we are in the situation considered in [22]; in particular there exists a closed holomorphic 1-form $\Omega$, non-singular and defining $\mathcal{F}$ on $M$, and we may describe the dynamics of $\mathcal{F}$ according to the group $\operatorname{Per}(\Omega) \subset(\mathbb{C},+)$ (cf. [22]). For instance, if $M$ is compact Kaehler with rank $H_{1}(M ; \mathbb{R}) \leq 2$ then $\mathcal{F}$ is a Seifert fibration (i.e., a foliation by compact leaves having finite holonomy groups).

2. Assume now that there is a flow $\varphi$ which is tangent to $\mathcal{F}$; this implies that given any $p \in M$ the leaf $L_{p}$ of $\mathcal{F}$ containing $p$ admits a holomorphic $\operatorname{map} \varphi_{p}: \mathbb{C} \rightarrow L_{p}$ and $L_{p}$ is holomorphically equivalent to one of the following Riemann surfaces: $\mathbb{C}, \mathbb{C}^{*}$ or a torus $\mathbb{C} / \mathbb{Z} \oplus \mathbb{Z}$. Moreover, $X(p)=\left.\frac{\partial \varphi_{t}}{\partial t}(p)\right|_{t=0}$ defines a global holomorphic vector field (possibly with singularities) on $M$, tangent to $\mathcal{F}$. If $X$ is singular but $M=\mathbb{C} P(2)$ then $X$ must be linear: $X=\lambda x \frac{\partial}{\partial x}+\mu y \frac{\partial}{\partial y}$ or $X=\lambda x \frac{\partial}{\partial x}+(x+\lambda y) \frac{\partial}{\partial y}$ in some affine chart $(x, y) \in \mathbb{C}^{2}$ and $\mathcal{F}$ is linear as well.

3 . Now we consider the non-singular case. If $M$ is compact and $X$ is nonsingular then the second Chern class of $M$ is zero: $c_{2}(M)=0$, and we may use the Enriques-Kodaira classification [1] in order to describe $M$. Actually, in [18] it is proved that such a surface must be one of the following: (1) a flat holomorphic fibre bundle over an elliptic curve with connected fibres; (2) a complex torus; (3) an elliptic surface without singular fibres or with singular fibres of type ${ }_{m} \mathrm{I}_{0}$ only; (4) a non-elliptic Hopf surface; (5) an Inoue surface of type $S_{N, p, q, r, t}^{(+)}$.

4. Finally, concerning the existence of compact leaves in the non-singular case we find that if $L_{0}$ is a toral leaf then we have two possibilities: (i) $L_{0}$ is an isolated toral leaf; (ii) $L_{0}$ is not an isolated toral leaf; in this case the holonomy group $\operatorname{Hol}\left(L_{0}\right) \hookrightarrow \operatorname{Diff}(\mathbb{C}, 0)$ is abelian with some finite pseudo-orbits so that it cannot contain non-trivial flat elements (cf. [7]) and therefore it is a finite group of rational rotations. Since $\operatorname{Hol}\left(L_{0}\right)$ is finite the global stability theorem in [2] implies that every leaf of $\mathcal{F}$ is compact with finite holonomy, therefore $\mathcal{F}$ is a Seifert fibration by complex tori.

2.2. Proof of Proposition 1. We shall keep the notation of the preceding subsection. The case where $\varphi_{t}$ is completely transverse to $\mathcal{F}$ in $\mathbb{C} P(2) \backslash \Gamma$ follows from [22]. Indeed, as we have seen above the foliation in $U=\mathbb{C} P(2) \backslash$ $\Gamma$ is given by a closed holomorphic one-form $\Omega$. Since the singularities of $\mathcal{F}$ in $\Gamma$ are hyperbolic, $\Omega$ extends meromorphically with simple poles along $\Gamma$ to a neighborhood of each singular point $p \in \operatorname{sing}(\mathcal{F}) \in \Gamma$ [21]. Thus we conclude by the Hartogs Theorem that $\Omega$ extends to a meromorphic one-form $\widetilde{\Omega}$ in $\mathbb{C} P(2)$. This one-form has simple poles in $\mathbb{C} P(2)$ and by the 
Integration Lemma is logarithmic; by [3] the foliation must be linear in some affine chart due to the fact that its singularities are hyperbolic.

Assume now that the flow is everywhere tangent to $\mathcal{F}$ in $U$. This means that the leaves of $\left.\mathcal{F}\right|_{U}$ are the nonsingular orbits of the vector field $X(p)=$ $\left.\left(\partial \varphi_{t}(p) / \partial t\right)\right|_{t=0}$. In particular $\left.\mathcal{F}\right|_{U}$ and therefore $\mathcal{F}$ is parabolic; since $\mathcal{F}$ has only hyperbolic singularities in $\mathbb{C} P(2)$, it follows from [4] that it is linear in some affine chart in $\mathbb{C}^{2}$.

Finally, assume that $\varphi_{t}$ is not completely transverse to $\mathcal{F}$ in $\mathbb{C} P(2) \backslash \Gamma$ but $\mathcal{F}$ is not the foliation induced by the vector field $X$ as above. If $L$ is a leaf of $\mathcal{F}$ in $U$ which is also invariant under $X$ then $L$ is covered by $\mathbb{C}$. If $L$ is non-algebraic then $\mathcal{F}$ has a transcendental leaf covered by $\mathbb{C}$ and according to [5] and [9] it follows that $\mathcal{F}$ admits a non-trivial holonomy invariant measure; moreover, $[9], \mathcal{F}$ is a linear hyperbolic foliation due to its hyperbolic singularities. If the closure $\bar{L} \subset \mathbb{C} P(2)$ is an algebraic curve, say $\Gamma^{\prime}$, then $U^{\prime}=\mathbb{C} P(2) \backslash\left(\Gamma \cup \Gamma^{\prime}\right)$ is also affine. Due to the hyperbolic singularities $\mathcal{F}$ admits no rational first integral and therefore by a theorem of Darboux [13], $\mathcal{F}$ has only finitely many algebraic invariant curves. This reduces the proof to the two cases above. In other words, we may assume that either $\mathcal{F}$ is completely transverse to $X$ or it coincides with the foliation of $X$ in $\mathbb{C} P(2) \backslash \Gamma$. In either case the foliation is linear in some affine chart in $\mathbb{C}^{2}$.

\section{Entire automorphisms - Proof of Lemma 1 and Theorem 1.} Let $\mathcal{F}$ be an algebraic foliation on $\mathbb{C}^{2}$. Then $\mathcal{F}$ admits an extension of algebraic type to a foliation (also denoted by) $\mathcal{F}$ on $\mathbb{C P}(2)=\mathbb{C}^{2} \cup \mathbb{P}_{\infty}^{1}$. Let $\varphi: \mathbb{C}^{2} \rightarrow \mathbb{C}^{2}$ be an entire (algebraic or transcendental) automorphism of $\mathbb{C}^{2}$ that preserves $\mathcal{F}$. Under which conditions on $\mathcal{F}$ is $\varphi$ algebraic?

3.1. Case of $\mathbb{P}_{\infty}^{1}$ is not $\mathcal{F}$-invariant-Proof of Extension Lemma 1. Assume that $\mathbb{P}_{\infty}^{1}$ is not $\mathcal{F}$-invariant; the set of points $p \in \mathbb{P}_{\infty}^{1}$ such that $\mathcal{F}$ is not transverse to $\mathbb{P}_{\infty}^{1}$ in a neighborhood of $p$ is a finite subset of $\mathbb{P}_{\infty}^{1}$, say $T\left(\mathcal{F}, \mathbb{P}_{\infty}^{1}\right)=\left\{p_{1}, \ldots, p_{r}\right\}$. Let $p \in \mathbb{P}_{\infty}^{1} \backslash T\left(\mathcal{F}, \mathbb{P}_{\infty}^{1}\right)$ be given and choose a flow-box neighborhood $U$ of $p$ in $\mathbb{C} P(2)$ such that in local coordinates $(x, y)$ in $U$ we have $x(p)=y(p)=0, \mathbb{P}_{\infty}^{1} \cap U=\{x=0\}$ and $\left.\mathcal{F}\right|_{U}: d y=0$. Also we may assume that $\{y=0\} \subset L_{p}$. Denote by $L_{p}$ the leaf of $\mathcal{F}$ that contains $p$ and by $L_{p}^{*}=L_{p} \backslash \mathbb{P}_{\infty}^{1}$ the corresponding leaf of $\left.\mathcal{F}\right|_{\mathbb{C}^{2}}$. Since $\varphi: \mathbb{C}^{2} \rightarrow \mathbb{C}^{2}$ is an automorphism that preserves $\mathcal{F}$ we have $L_{p}^{*}=\varphi\left(L^{*}\right)$, where $L^{*}=L \backslash \mathbb{P}_{\infty}^{1} \subset \mathbb{C}^{2}$ for some leaf $L$ of $\mathcal{F}$ on $\mathbb{C} P(2)$. Now, using the flow-box in $U$ we may choose a disk $\Sigma \subset U \cap \mathbb{C}^{2}$ transverse to $\mathcal{F}$ with the properties that each plaque of $\left.\mathcal{F}\right|_{U}$ cuts $\Sigma$ at most once and the same holds for the disk $\mathbb{P}_{\infty}^{1} \cap U=: \Sigma_{\infty}$. 
Let $p^{\prime} \in \Sigma \cap L_{p}, p^{\prime} \in\{y=0\}$; choose a simple path $\alpha:[0,1] \rightarrow L_{p} \cap U$ such that $\alpha(t) \in\{y=0\}$ for all $t \in[0,1], \alpha(0)=p^{\prime}$ and $\alpha(1)=p$, and denote by $h_{\alpha}:\left(\Sigma, p^{\prime}\right) \rightarrow\left(\Sigma_{\infty}, p\right)$ the holonomy map corresponding to $\alpha$. Let $\Sigma_{1}$ be the inverse image $\Sigma_{1}=\varphi^{-1}(\Sigma) \subset \mathbb{C}^{2}$. Then $\Sigma_{1}$ is also a transverse disk to $\mathcal{F}$ in $\mathbb{C}^{2}$ and $\Sigma_{1} \cap L \ni p_{1}^{\prime}=\varphi^{-1}\left(p^{\prime}\right)$. Now we choose any simple path $\beta:[0,1] \rightarrow L$ with the following properties: $\beta(0)=p_{1}$ and $\beta(1)=q^{\prime} \in \mathbb{C}^{2}$ is a point which belongs to a flow-box $V \subset \mathbb{C P}(2)$ centered at a point $q \in \mathbb{P}_{\infty}^{1} \cap L$.

Fig. 1

In particular we may assume that $q^{\prime}$ and $q$ belong to the same plaque $P$ of $\left.\mathcal{F}\right|_{V}$, which is a disk $P \approx \mathbb{D}$. We fix a transverse disk $\Sigma_{2} \subset V$ to $\mathcal{F}$ with $\Sigma_{2} \cap P=q^{\prime}$ and choose any simple path $\alpha_{1}:[0,1] \rightarrow P$ with $\alpha_{1}(0)=q^{\prime}$, $\alpha_{1}(1)=q$.

Denote by $h_{\beta}:\left(\Sigma_{1}, p_{1}^{\prime}\right) \rightarrow\left(\Sigma_{2}, q^{\prime}\right)$ and $h_{\alpha_{1}}:\left(\Sigma_{2}, q^{\prime}\right) \rightarrow\left(\Sigma_{\infty}^{\prime}, q\right)$ the holonomy diffeomorphisms corresponding to $\beta$ and $\alpha_{1}$ respectively, where $\Sigma_{\infty}^{\prime}=\mathbb{P}_{\infty}^{1} \cap V$ is transverse to $\mathcal{F}$. 
Given a point $a \in \Sigma_{\infty}$ close enough to $p$, we define $\varphi_{\beta}^{-1}(a):=h_{\alpha_{1}} \circ h_{\beta}$ $\circ h_{\alpha}^{-1}(a)$.

Lemma 2. If the holonomy of $L$ is trivial then $\varphi_{\beta}^{-1}$ does not depend on the choice of $\beta$.

Proof. This is clear.

Proof of Lemma 1. We keep the above notation. Since $\left.\mathcal{F}\right|_{U}$ and $\left.\mathcal{F}\right|_{V}$ are trivial it follows that for a leaf $L$ with trivial holonomy as above $\varphi_{\beta}^{-1}$ is an extension of $\varphi^{-1}$ to $\Sigma_{\infty}=U \cap \mathbb{P}_{\infty}^{1}$ and by the Hartogs Extension Theorem $\varphi^{-1}$ extends to a meromorphic map $\bar{\varphi}^{-1}: \mathbb{C} P(2) \rightarrow \mathbb{C} P(2)$. Since the same holds for $\varphi$ and such extensions must clearly satisfy $\bar{\varphi} \circ \bar{\varphi}^{-1}=$ $\operatorname{Id}_{\mathbb{C} P(2)}$ we conclude that $\varphi$ extends to a bimeromorphism $\bar{\varphi}$ of $\mathbb{C} P(2)$; the bimeromorphism $\bar{\varphi}$ still preserves $\mathcal{F}$ on $\mathbb{C} P(2)$. Using now the fact that for $\mathcal{F}$ arbitrary (with $\mathbb{P}_{\infty}^{1}$ not invariant) the set of leaves with trivial holonomy is residual we obtain Lemma 1.

3.2. Proof of Theorem 1. Let $\mathcal{F}$ be an algebraic foliation on $\mathbb{C} P(2)$ such that $\operatorname{sing}(\mathcal{F}) \cap \mathbb{P}_{\infty}^{1}$ is hyperbolic and $\operatorname{Aut}\left(\left.\mathcal{F}\right|_{\mathbb{C}^{2}}\right)$ contains a transcendental element $\varphi: \mathbb{C}^{2} \rightarrow \mathbb{C}^{2}$. According to Lemma 1 the line at infinity is invariant. Denote by $L_{\infty}=\mathbb{P}_{\infty}^{1} \backslash\left(\mathbb{P}_{\infty}^{1} \cap \operatorname{sing}(\mathcal{F})\right)$ the corresponding leaf of $\mathcal{F}$. If the holonomy group of $L_{\infty}$ is solvable then, according to [21] and [8], due to the fact that the singularities in $\operatorname{sing}(\mathcal{F}) \cap \mathbb{P}_{\infty}^{1}$ are hyperbolic, the foliation is either a Riccati foliation or a logarithmic foliation. Thus we may assume that $\operatorname{Hol}\left(\mathcal{F}, L_{\infty}\right)$ is a non-solvable group. Since by the Index Theorem $\operatorname{sing}(\mathcal{F}) \cap$ $\mathbb{P}_{\infty} \neq \emptyset$, the group $\operatorname{Hol}\left(\mathcal{F}, L_{\infty}\right)$ contains hyperbolic elements. Using now [19], [15], [9], [12], [23] we shall prove that $\mathcal{F}$ is chaotic:

\section{Lemma 3. In the situation above, $\mathcal{F}$ has no algebraic leaf $C \subset \mathbb{C}^{2}$.}

Proof. Assume that $\mathcal{F}$ has some algebraic invariant curve $C \subset \mathbb{C}^{2}$; denote by $L=C \backslash \operatorname{sing}(\mathcal{F})$ the corresponding leaf of $\left.\mathcal{F}\right|_{\mathbb{C}^{2}}$ (we may work with $C$ irreducible). The leaf $L$ is then a parabolic leaf of $\left.\mathcal{F}\right|_{\mathbb{C}^{2}}$; denote by $L^{\prime}=\varphi(L) \subset \mathbb{C}^{2}$ its image under $\varphi$. If $L^{\prime}$ is not algebraic (i.e., the closure $\overline{L^{\prime}} \subset \mathbb{C}^{2}$ is not an algebraic curve) then the intersection $L^{\prime} \cap D$ accumulates to the origin $o=D \cap L_{\infty}$ of any disk $D$ transverse to $\mathcal{F}$ and to $L_{\infty}$. Since $C$ is closed in $\mathbb{C}^{2}$ the same holds for $L^{\prime}$ and therefore it induces a pseudo-orbit in the holonomy group $\operatorname{Hol}\left(\mathcal{F}, l_{\infty}, D\right)$, which is closed off the origin $o \in D$. According to the density theorem in [19], $\operatorname{Hol}\left(\mathcal{F}, L_{\infty}, D\right)$ must be solvable, a contradiction. Thus necessarily $L^{\prime}$ is algebraic. If $\mathcal{F}$ has infinitely many algebraic leaves then by a theorem of Darboux [13], $\mathcal{F}$ is given by a rational map $R: \mathbb{C} P(2) \rightarrow \overline{\mathbb{C}}$; this is not compatible with the hyperbolic singularities. We may therefore assume that $\varphi(C)=C$. Now we recall the following notions from [14]: 
Let $C \subset \mathbb{C}^{2}$ be an irreducible algebraic affine curve. We say that $C$ is general if any analytic automorphism of $\mathbb{C}^{2}$ which transforms $C$ into an algebraic curve is algebraic.

TheOREM 3 (Kizuka [14]). Let $C \subset \mathbb{C}^{2}$ be an affine algebraic curve. Then $C$ is general provided that the closure $\bar{C}$ of $C$ on $\mathbb{C} P(2)$ intersects the line at infinity $\mathbb{P}_{\infty}^{1}$ at more than two points. If $C$ is not general then we have the following possibilities for $C$ after an algebraic affine change of coordinates:

(K.1) $C: \alpha(x)+y \beta(x)=0($ special case);

(K.2) $C$ is a sum of several prime surfaces of a monomial $f=x^{m} y^{n}$ where $m, n \in \mathbb{N}$

(K.3) $C$ is a sum of several prime surfaces of a polynomial $f=x^{m}\left(x^{l} y+\right.$ $\left.P_{l-1}(x)\right)^{n}$, where $P_{l-1}$ is a one-variable polynomial of degree at most $l-1$ with $P_{l-1}(0) \neq 0$, and $m, n \in \mathbb{N}$.

In each case above straightforward calculations show that $\mathcal{F}$ has either some separatrix at infinity which is not smooth at the singularity or some separatrix which is not transverse to the line at infinity. This contradicts the invariance of $\mathbb{P}_{\infty}^{1}$ and the hyperbolicity of the singularities in $\mathbb{P}_{\infty}^{1}$. Lemma 3 is now proved.

Now we may finish the proof of Theorem 1:

(i) $\left.\mathcal{F}\right|_{\mathbb{C}^{2}}$ has no parabolic leaf: otherwise according to [16], [4], [21], $\left.\mathcal{F}\right|_{\mathbb{C}^{2}}$ would have some algebraic leaf, contradicting Lemma 3.

(ii) By (i) the leaves of $\left.\mathcal{F}\right|_{\mathbb{C}^{2}}$ are hyperbolic (in the sense of potential theory) and covered by the disk $\mathbb{D}$. Moreover, the leaves of $\left.\mathcal{F}\right|_{\mathbb{C}^{2}}$ have exponential growth (otherwise, according to $[9], \mathcal{F}$ would admit some holonomy invariant measure for the holonomy group of $L_{\infty}=\mathbb{P}_{\infty}^{1}$ and this measure would imply that the holonomy group $\operatorname{Hol}\left(\mathcal{F}, L_{\infty}\right)$ is solvable, giving a contradiction).

(iii) Since the holonomy group of $\mathbb{P}_{\infty}^{1}$ is non-solvable and contains hyperbolic elements, the foliations $\left.\mathcal{F}\right|_{\mathbb{C}^{2}}$ and $\mathcal{F}$ on $\mathbb{C} P(2)$ are topologically rigid and, as follows from the techniques in [15], we may also conclude that $\left.\mathcal{F}\right|_{\mathbb{C}^{2}}$ and $\mathcal{F}$ on $\mathbb{C} P(2)$ are ergodic.

(iv) The density of the leaves in $\mathbb{C}^{2}$ is proved as in [15] as a consequence of the density results in [19] and [23] for non-solvable groups of germs of onedimensional complex diffeomorphisms at the origin. Theorem 1 is therefore proved.

4. Case of an algebraic invariant curve in $\mathbb{C}^{2}$ - Theorem 2. Let $\mathcal{F}$ be an algebraic foliation on $\mathbb{C} P(2)$ such that $\operatorname{Aut}\left(\left.\mathcal{F}\right|_{\mathbb{C}^{2}}\right)$ contains a transcendental element $\varphi: \mathbb{C}^{2} \rightarrow \mathbb{C}^{2}$ and having an invariant algebraic curve 
$C \subset \mathbb{C}^{2}$. As in the proof of Theorem 1 denote by $L=C \backslash \operatorname{sing}(\mathcal{F})$ the corresponding leaf of $\left.\mathcal{F}\right|_{\mathbb{C}^{2}}$ and by $L^{\prime}=\varphi(L) \subset \mathbb{C}^{2}$ its image under $\varphi$.

4.1. If $L^{\prime}$ is not algebraic (i.e., the closure $\overline{L^{\prime}} \subset \mathbb{C}^{2}$ is not an algebraic curve) then by the Darboux Theorem [13], $\mathcal{F}$ has only a finite number of algebraic leaves; we may therefore assume that $L^{\prime}$ is a transcendental leaf of finite type and properly embedded so that, by the Corollary in [3], necessarily $L^{\prime}$ (and therefore $L$ ) is isomorphic to $\mathbb{C}$ and also $\left.\mathcal{F}\right|_{\mathbb{C}^{2}}$ is holomorphically equivalent to the foliation $d y=0$ on $\mathbb{C}^{2}$. In this case, up to some entire automorphism, we have $\varphi(x, y)=(a(x, y), b(y))$ and therefore $\varphi(x, y)=\left(a_{0}(y) x+a_{1}(y), b_{0}+b_{1} y\right)$ for some $b_{0}, b_{1} \in \mathbb{C}$, $b_{1} \neq 0, a_{0}(y), a_{1}(y) \in \mathcal{O}(\mathbb{C})$, with $a_{0}(y) \neq 0$ for all $y \in \mathbb{C}$. Again we may find holomorphic coordinates on $\mathbb{C}^{2}$ such that $\left.\mathcal{F}\right|_{\mathbb{C}^{2}}: d y=0$ and $\varphi(x, y)=\left(e^{u(y)} x+v(y), y\right)$ for some holomorphic $u, v \in \mathcal{O}(\mathbb{C})$.

EXAmple 1. Let us see an example of the above situation: $X(x, y)=$ $(1+x y) \frac{\partial}{\partial x}-y^{2} \frac{\partial}{\partial y}$ generates a foliation $\mathcal{F}$ on $\mathbb{C}^{2}$ with the entire first integral $f(x, y)=y e^{x y}$. The algebraic curve $C:\{y=0\}$ is $\mathcal{F}$-invariant and we have an entire non-algebraic automorphism $\varphi(x, y)=\left(x e^{P(x, y)}, y e^{x y}\right)$ for some polynomial $P(x, y)$ (see [24]) and clearly $\varphi$ preserves $\mathcal{F}$. The vector field $X$ is not complete.

4.2. Suppose now $C$ is $\mathcal{F}$-invariant, algebraic and fixed by $\varphi$. According to Kizuka's Theorem above [14], $\varphi$ is algebraic or, up to polynomial automorphism, $C$ belongs to the following list:

(K.1) $\alpha(x)+y \beta(x)=0$, where $\alpha$ and $\beta$ are one-variable polynomials;

(K.2) a union of irreducible components of fibers of $x^{n} y^{m}$, where $n, m \in \mathbb{N}$ satisfy $\langle n, m\rangle=1$;

(K.3) a union of irreducible components of fibers of a polynomial $Q$ of type $Q(x, y)=x^{n}\left(x^{l} y+P(x)\right)^{m}$, with $n, m, l \in \mathbb{N},\langle n, m\rangle=1, P$ is a one-variable polynomial of degree at most $l-1, P(0) \neq 0$.

In any of the cases (K.1), (K.2) and (K.3) above the irreducible components of the projective curve $\bar{C} \subset \mathbb{C} P(2)$ are rational and we have $\sharp \bar{C} \cap \mathbb{P}_{\infty}^{1}$ $\leq 2$; in particular, each irreducible component of $C$ is diffeomorphic either to $\mathbb{C}$ or to $\mathbb{C}^{*}=\mathbb{C} \backslash\{0\}$. Examples of this situation are given by vector fields of the form

$$
\begin{aligned}
X= & x\left(\mu+m C_{1}\right) \frac{\partial}{\partial x} \\
& -\left\{\frac{\left(x^{l} y+P(x)\right)\left(\lambda+n C_{1}\right)}{x^{l}}+\frac{\left(l x^{l} y+x P^{\prime}(x)\right)\left(\mu+m C_{1}\right)}{x}\right\} \frac{\partial}{\partial y}
\end{aligned}
$$

with $C_{1}=x^{n}\left(x^{l} y+P(x)\right)^{m} \cdot \tau^{\prime}\left(x^{n}\left(x^{l} y+P(x)\right)^{m}\right)$. These are complete vector fields (see [11]) whose flow maps $\varphi_{t}: \mathbb{C}^{2} \rightarrow \mathbb{C}^{2}$ are not algebraic and preserve 
both the foliation $\left.\mathcal{F}_{X}\right|_{\mathbb{C}^{2}}$ and the algebraic invariant curve $C:\left\{x^{n}\left(x^{l} y+\right.\right.$ $\left.P(x))^{m}=0\right\}$.

4.3. Assume that $\left.\mathcal{F}\right|_{\mathbb{C}^{2}}$ has some irreducible algebraic invariant curve $C$ whose image $\varphi(C)$ is algebraic but is not $C$. Denote by $C_{\text {alg }}(\mathcal{F})$ the union of all irreducible algebraic curves $C_{1} \subset \mathbb{C}^{2}$ which are invariant under $\mathcal{F}$. We may assume that for any irreducible curve $C_{1} \in C_{\text {alg }}(\mathcal{F})$ we have $\varphi\left(C_{1}\right) \in$ $C_{\text {alg }}(\mathcal{F})$ but $\varphi\left(C_{1}\right) \neq C_{1}$. Then in particular we have $\varphi^{n}(C) \neq \varphi^{m}(C)$ for all $n \neq m$ and thus $\sharp C_{\text {alg }}(\mathcal{F})=+\infty$. By the Darboux Theorem [13], $\mathcal{F}$ admits a rational first integral $R: \mathbb{C}^{2} \rightarrow \overline{\mathbb{C}}$ and therefore $\mathcal{F}$ is a pencil of curves $\mathcal{F}:\{\lambda P+\mu Q=0\},(\lambda ; \mu) \in \mathbb{P}^{1}$, for some $P, Q \in \mathbb{C}[x, y]$.

It remains therefore to consider the following case:

4.4. $\left.\mathcal{F}\right|_{\mathbb{C}^{2}}$ has no algebraic leaf, and $\mathbb{P}_{\infty}^{1}$ is $\mathcal{F}$-invariant. This case contradicts the hypothesis of existence of the invariant curve $C$. Theorem 2 is therefore proved.

EXAMPLE 2. The linear foliation $\mathcal{F}_{\lambda}$ given on $\mathbb{C}^{2} \subset \mathbb{C} P(2)$ by $\mathcal{F}_{\lambda}$ : $x d y-\lambda y d x=0, \lambda \in \mathbb{C} \backslash\{0\}$, admits the following subgroup of automorphisms: $\left\{\varphi: \mathbb{C}^{2} \rightarrow \mathbb{C}^{2} ; \varphi(x, y)=\left(x e^{w}, c y e^{\lambda w}\right)\right.$, where $\left.w \in \mathbb{C}, c \in \mathbb{C}^{2}\right\}$. Conversely, for $\lambda \notin \mathbb{Q}$, up to conjugacy with $\sigma(x, y)=(y, x)$, these are the only automorphisms of $\mathcal{F}_{\lambda}$ in $\mathbb{C}^{2}$ : given $\varphi: \mathbb{C}^{2} \rightarrow \mathbb{C}^{2}$ preserving $\mathcal{F}_{\lambda}$ and the coordinate axis we may write $\varphi(x, y)=(x . u, y . v)$ for entire non-vanishing functions $u, v$ on $\mathbb{C}^{2}$. Then a straightforward calculation shows that $\varphi^{*}\left(\mathcal{F}_{\lambda}\right)=\mathcal{F}_{\lambda}$ iff $v u^{-\lambda} \in \mathcal{O}\left(\mathbb{C}^{2}\right)$ is an entire first integral for $\mathcal{F}_{\lambda}$ iff $v u^{-\lambda} \equiv$ const. If we write $u=e^{w}$ then we obtain $\varphi(x, y)=\left(x . e^{w}, c . y e^{\lambda w}\right)$ for $w \in \mathcal{O}\left(\mathbb{C}^{2}\right)$. Such a map is a foliation automorphism iff $w \in \mathbb{C}$ is constant.

Examples of transcendental foliation automorphisms are given by complete polynomial vector fields having non-polynomial flows (cf. [11]).

\section{References}

[1] W. Barth, C. Peters and A. Van de Ven, Compact Complex Surfaces, Springer, Berlin, 1984.

[2] M. Brunella, A global stability theorem for transversely holomorphic foliations, Ann. Global Anal. Geom. 15 (1997), 179-186.

[3] -, Sur les courbes intégrales propres des champs de vecteurs polynomiaux, Topology 37 (1998), 1229-1246.

[4] - Foliations on the complex projective plane with many parabolic leaves, Ann. Inst. Fourier (Grenoble) 44 (1994), 1237-1242.

[5] -, Courbes entières et feuilletages holomorphes, Enseign. Math. 45 (1999), 195-216.

[6] -, Minimal models of foliated algebraic surfaces, Bull. Soc. Math. France 127 (1999), 289-305.

[7] C. Camacho, On the local structure of conformal mappings and holomorphic vector fields in $\mathbb{C}^{2}$, Astérisque 59-60 (1978), 83-94. 
[8] C. Camacho and B. Scárdua, Holomorphic foliations with Liouvillian first integrals, Ergodic Theory Dynam. Systems 21 (2001), 717-756.

[9] J. Canille and B. Scárdua, On the growth of complex projective foliations, Internat. J. Math. 13 (2002), 695-726.

[10] S. Cantat and C. Favre, Symétries birationnelles de surfaces feuilletées, J. Reine Angew. Math., to appear.

[11] D. Cerveau and B. Scárdua, Complete polynomial vector fields in two complex variables, Forum Math., to appear.

[12] Yu. S. Il'yashenko, Density of an individual solution and ergodicity of a family of solutions of the equation $d \eta / d \xi=P(\xi, \eta) / Q(\xi, \eta)$, Mat. Zametki 4 (1968), 741-750 (in Russian).

[13] J.-P. Jouanolou, Équations de Pfaff algébriques, Lecture Notes in Math. 708, Springer, Berlin, 1979.

[14] T. Kizuka, Analytic automorphisms and algebraic automorphisms of $\mathbb{C}^{2}$, Tôhoku Math. J. 31 (1979), 553-565.

[15] A. Lins Neto, P. Sad and B. Azevedo Scárdua, On topological rigidity of projective foliations, Bull. Soc. Math. France 126 (1998), 381-406.

[16] M. McQuillan, Diophantine approximation and foliations, Publ. Math. I.H.E.S. 87 (1998), 121-174.

[17] L. G. Mendes, Kodaira dimension of singular holomorphic foliations, $\mathrm{PhD}$ thesis at IMPA, preprint, 1999.

[18] A. Mizuhara, On compact complex analytic surfaces admitting nonvanishing holomorphic vector fields, Tensor 32 (1978), 101-106.

[19] I. Nakai, Separatrices for nonsolvable dynamics on $\mathbb{C}, 0$, Ann. Inst. Fourier (Grenoble) 44 (1994), 569-599.

[20] J. V. Pereira and P. Sánchez, Transformation groups of holomorphic foliations, Comm. Anal. Geom. 10 (2002), 1115-1123.

[21] B. Scárdua, A remark on parabolic projective foliations, Hokkaido Math. J. 28 (1999), 231-252.

[22] - , Holomorphic Anosov systems, fibrations and Kähler manifolds, Dynamical Systems, to appear.

[23] A. A. Shcherbakov, On the density of the orbit of a pseudogroup of conformal mappings and generalization of the Khudaü-Verenov theorem, Moscow Univ. Math. Bull. 37 (1982), no. 4, 10-16; transl. from Vestnik Moskov. Univ. Ser. 1982, no. 4, 10-15.

[24] H. Shiga, On the automorphism of $\mathbb{C}^{2}$ with invariant axes, J. Math. Soc. Japan 29 (1977), 529-535.

Instituto de Matemática

Universidade Federal do Rio de Janeiro

C.P. 68530

CEP. 21945-970 Rio de Janeiro, RJ, Brazil

E-mail: scardua@im.ufrj.br 